\title{
Multiplication et différenciation des cellules adipeuses
}

Les étapes de la différenciation adipocytaire sont maintenant bien caractérisées in vitro ; les deux principaux stades en sont les suivants : 1) des précurseurs ne se divisant plus expriment des marqueurs précoces de différenciation ; 2) sous l'action de stimuli hormonaux, principalement de l'hormone de croissance, ces précurseurs se divisent un petit nombre de fois et se transforment en adipocytes adultes. In vivo, il semble que l'acquisition de nouveaux adipocytes provienne de la différenciation des précurseurs situés dans le stromavasculaire du tissu adipeux.

\section{Gérard Ailhaud}

Professeur de biochimie. Directeur de laboratoire au Cnrs

\section{ADRESSE}

G. Ailhaud : laboratoire de biologie du développement du tissu adipeux, centre de biochimie (Cnrs LP 7300), faculté des sciences, parc 'étude de la multiplication et de la différenciation des cellules adipeuses est au cœur des problèmes posés par le développement normal et pathologique du tissu adipeux. Il n'est pas inutile de rappeler que ce tissu est physiologiquement important comme réservoir de triglycérides, source d'énergie, ainsi que de cholestérol non estérifié. Chez la femme, il constitue en outre une source non négligeable de production d'estrogènes à partir d'androstènedione.

Le développement excessif de la masse adipeuse est très généralement lié à une hypertrophie cellulaire*. Dans le cas des obésités les plus sévères, chez l'animal comme chez l'homme, cette hypertrophie paraît s'accompagner d'une hyperplasie** significative. Il faut toutefois noter que la cellularité du tissu adipeux, c'est-àdire la détermination du nombre de cellules adipeuses, reste tech-

\footnotetext{
* Hypertrophie cellulaire : augmentation du volume des cellules et du contenu en triglycérides.

** Hyperplosie : augmentation du nombre des cellules.
}

niquement imprécise. En ce qui concerne l'hypertrophie, rappelons que la perte de poids consécutive à un traitement non chirurgical (régime, jeûne même prolongé) n'est liée qu'à une diminution du contenu cellulaire moyen en triglycérides, sans diminution détectable du nombre de cellules adipeuses [1]. Le processus est pleinement réversible quelques semaines après l'interruption du traitement chez l'animal, qui retrouve alors sa masse adipeuse initiale.

L'extrapolation de telles observations au traitement des obésités humaines permet de comprendre les échecs médicaux constatés après interruption d'un régime. L'élimination d'une masse adipeuse par la technique dite de "lipo-aspiration" ne représente sans doute pas. non plus une panacée si l'on se souvient que, après lipectomie chez le rat, ont été démontrées à la fois une augmentation (compensatoire ?) du nombre d'adipocytes dans les sites adipeux restés intacts et une régénération des adipocytes à partir de cellules précurseurs dans le site en principe éradiqué [2]. Contraire- 
ment à des affirmations dogmatiques antérieures, et bien que les adipocytes pleinement différenciés ne se divisent plus in vivo, il est maintenant démontré qu'une augmentation du nombre des adipocytes à partir de cellules précurseurs persiste même à l'âge adulte chez diverses espèces ( $\mathrm{y}$ compris chez l'homme) $[2,3]$; cette augmentation est variable selon le site adipeux considéré et en fonction des conditions nutritionnelles.

$\mathrm{La}$ caractérisation des facteurs impliqués dans la multiplication des cellules précurseurs et leur différenciation en adipocytes se révèle donc indispensable mais compliquée in vivo en raison de la multiplicité des signaux biologiques potentiels, suggérée par la multiplicité des causes (nutritionnelles et génétiques, entre autres) conduisant à un développement excessif du tissu adipeux. Dans la perspective d'une approche plus directe, la dernière décennie $\mathrm{a}$ vu se développer de nombreux modèles cellulaires in vitro destinés à mieux caractériser ces facteurs et à analyser le processus de différenciation adipocytaire $[4,5]$.

\section{Les modèles cellulaires}

La culture de cellules adipeuses a réellement pris son essor à partir de 1974 grâce aux travaux de H. Green et de ses associés. Les premières cellules caractérisées comme précurseurs de cellules adipeuses ont été obtenues en lignée établie à partir d'embryon total de souris [6], puis à partir du tissu adipeux de la même espèce [7]. Depuis lors, de nombreuses autres lignées préadipocytaires de rongeurs ont été établies. Des cellules précurseurs d'adipocytes provenant de diverses espèces peuvent être également obtenues en culture primaire à partir de la fraction stromavasculaire du tissu adipeux, c'està-dire après dissociation des cellules composant le tissu adipeux et élimination des adipocytes mûrs, ainsi que de la majorité des cellules contaminantes (cellules endothéliales, mastocytes) (Tableau I, figure 1). La culture de cellules précurseurs d'adipocytes humains vient de faire un progrès sensible. En cultivant la fraction stromavasculaire du tissu adipeux inguinal de jeune enfant dans un milieu chimiquement défini, on peut obtenir des cellules possédant des enzymes caractéristiques des adipocytes mûrs (lipoprotéine lipase et glycérophosphate déshydrogénase) et accumulant des triglycérides [8]. Signalons à ce propos que l'adipocyte mûr, c'està-dire pleinement différencié, ne possède aucun marqueur phénotypique qui lui soit propre. C'est donc l'ensemble des différents marqueurs qui peut être considéré comme caractéristique de la cellule adipeuse (figure 2).

\section{Polyclonalité du tissu adipeux}

La notion de polyclonalité du tissu adipeux, récemment étayée, semble importante pour comprendre l'acquisition permanente de nouveaux adipocytes dans certains sites adipeux. En effet, dans le cas bien étudié du tissu adipeux périrénal de rat, il apparaît que la population de cellules précurseurs d'adipocytes en culture primaire est constituée de clones cellulaires qui diffèrent dans leur capacité à se différencier in vitro et que, pour un même site adipeux, cette capacité diminue avec l'âge [9-11]. On peut imaginer que la capacité à se différencier des cellules précurseurs restantes pourrait être différente d'un site adipeux à un autre selon la proportion de clones cellulaires recrutés antérieurement au cours du développement. Cette hypothèse permettrait de rendre compte de l'hyperplasie limitée à quelques sites qui peut être constatée chez divers mammiferres, y compris dans l'espèce humaine en fonction du sexe. L'acquisition de nouvelles cellules adipeuses pọse en fait très directement le problème de la caractérisation de stimuli capables de faire engager les cellules précurseurs d'adipocytes dans leur programme de différenciation. Pour l'essentiel, ce p:oblème a fait l'objet de travaux intensifs sur les préadipocytes provenant de lignées établies.

\section{Différenciation précoce et terminale}

In vitro, le processus de différenciation adipocytaire comporte clairement des événements précoces

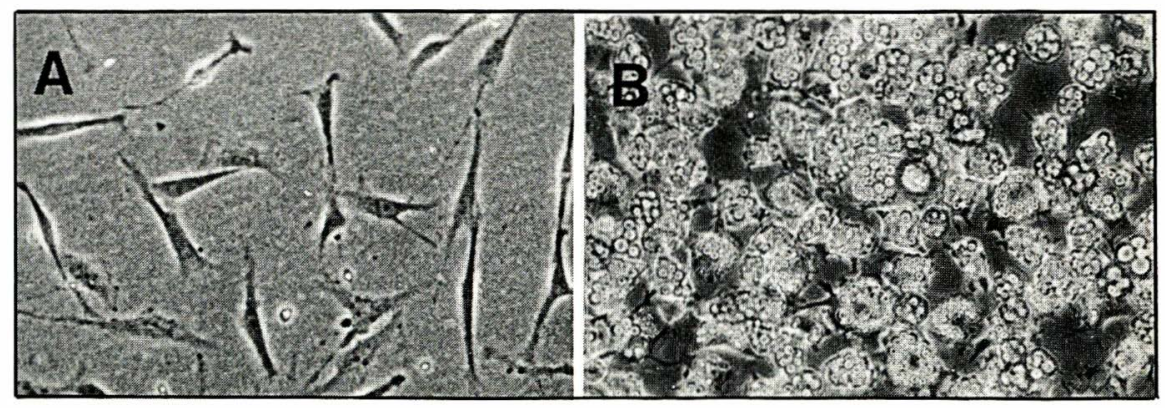

Figure 1. Changements morphologiques des cellules au cours de la différenciation adipocytaire. Ces clichés montrent le développement morphologique de cellules présentes dans la fraction stroma-vasculaire de tissu périépididymaire de rat âgé de quatre semaines. Les cellules sont maintenues dans un milieu de culture dépourvu de sérum et chimiquement défini [11]. In vivo comme in vitro (A), les cellules précurseurs présentent pendant la phase de prolifération une morphologie fibroblastique.

Par la suite, elles s'arrondissent et accumulent des triglycérides sous une forme multiloculaire (B). La formation d'adipocytes uniloculaires peut être observée si l'on enrichit le milieu de culture en triglycérides. 


\section{RÉFÉRENCES}

1. Miller WH Jr, Faust IM, Goldberger AC, Hirsch J. Effects of severe long-term deprivation and refeeding on adipose tissue cells in the rat. Am $J$ Physiol 1983 ; 245 : E74-E80.

2. Faust IM, Miller WH. Hyperplastic growth of adipose tissue in obesity. In : Angel A, Hollenberg CH, Roncari DAK, eds. The Adipocyte and Obesity: Cellular and Molecular Mechanisms. New York: Raven Press, 1981 ; 41-51.

3. Faust IM, Johnson PR, Stern JS, Hirsch J. Diet-induced adipocyte number increase in adult rats : a new model of obesity. $A m J$ Physiol 1978 ; 235 : E275-86.

4. Green H. Adipose conversion : a program of differentiation. In : Ailhaud G, ed. Obesity Cellular and Molecular Aspects. Paris : Éditions INSERM, $1979:$ 15-24.

5. Ailhaud G. Adipose cell differentiation in culture. Mol Cell Biochem 1982; $49: 17-31$.

6. Green H, Kehinde O. Sublines of mouse 3 T3 cells that accumulate lipid. Cell $1974 ; 1$ : 113-6.

7. Négrel R, Grimaldi P, Ailhaud G. Establishment of preadipocyte clonal line from epididymal fat pad of ob/ob mouse that responds to insulin and to lipolytic hormones. Proc Natl Acad Sci USA 1978; 75 : 6054-8.

8. Deslex S, Négrel R, Vannier C, Étienne J, Ailhaud G. Differentiation of human adipocyte precursors in a chemically defined serum-free medium. Int $J$ Obes $1986 ; 10$ : 19-27.

9. Björntorp $\mathbf{P}$, Karlsson $\mathbf{M}$, Pettersson $\mathbf{P}$, Sypniewska G. Differentiation and function of rat adipocyte precursor cells in primary culture. J Lipid Res 1980 ; 21 : 714-23.

10. Djian P, Roncari DAK, Hollenberg CH. Adipocyte precursor clones vary in capacity for differentiation. Metabolim 1985 ; 34 : 880-3.

11. Deslex S, Négrel R, Ailhaud G. Development of a chemically defined serum-free medium for differentiation of rat adipose precursor cells. Exp Cell Res 1987; 168: 15-30.

12. Amri E, Dani C, Doglio A, Grimaldi P, Ailhaud G. Coupling of growth arrest and expression of early markers during adipose conversion of preadipocyte cell lines. Biochem Biophys Res Commun 1986 ; 137 : 903-10.

13. Pairault J, Green H. A study of the adipose conversion of suspended $3 \mathrm{~T} 3$ cells by using glycerophosphate dehydrogenase as differentiation marker. Proc Natl Acad Sci USA et des événements tardifs. Le démarrage du programme de différenciation (figure 2) paraît uniquement lié à l'arrêt des cellules en phase $G_{1}$ du cycle cellulaire et se caractérise par l'émergence de marqueurs comme la lipoprotéine lipase, enzyme-clef in vivo de l'assimilation des triglycérides circulants (sous forme de lipoprotéines) par le tissu adipeux, et l'ARN messager pOb24 de $6 \mathrm{~kb}$ [12] ; recherché chez la souris adulte, ce messager semble spécifique du tissu adipeux, où il est uniquement retrouvé dans la fraction stroma-vasculaire contenant les cellules précurseurs d'adipocytes. Contrairement à l'émergence des marqueurs précoces, celle des marqueurs plus tardifs est dépendante de la présence de plusieurs hormones (voir ci-dessous) et elle est précédée de mitoses en nombre très restreint. L'entrée des cellules contenant les marqueurs précoces en phase $\mathrm{S} d u$ cycle cellulaire, caractérisée par la synthèse d'ADN, s'accompagne d'une ou deux mitoses, puis de l'apparition de la glycérophosphate déshydrogénase et de lipides neutres. Cette prolifération limitée correspond en fait à une expansion clonale de cellules en voie de différenciation terminale [12-14]. Basée sur l'ensemble des données disponibles, la chronologie d'apparition des phénotypes (figure 2) indique que la diminution de capacité d'oxydation des acides gras ainsi que l'augmentation de la réponse aux hormones lipolytiques (mesurée par la production d'AMPc) et à l'insuline seraient contemporaines de l'expression des marqueurs précoces. L'expression des marqueurs tardifs comporte trois étapes successives : au cours de la première étape, les cellules acquièrent la machinerie enzymatique nécessaire à la synthèse des acides gras et du glycérophosphate, précurseur tricarboné des triglycérides. C'est au cours de cette étape que sont exprimés la phosphodiestérase très affine pour l'AMP cyclique et la lipase sensible aux hormones, enzyme responsable de la dégradation des triglycérides intracellu- laires, ainsi que l'ARN messager codant pour une protéine aP2 de $13 \mathrm{kD}$ (kilodaltons) spécifique du tissu adipeux et sans doute impliquée dans le transport intracellulaire des acides gras [15]. La mise en place de la réponse lipolytique des cellules adipeuses est alors achevée. La seconde étape succède très rapidement à la première et correspond à l'acquisition des enzymes nécessaires à l'activation des acides gras et à leur estérification en triglycérides [16]. L'accumulation de triglycérides, visible au microscope, se produit à ce moment-là et précède une troisième étape qui voit l'émergence de l'ARN messager codant pour la phosphoénolpyruvate carboxykinase et de deux espèces d'un ARN messager supposé coder pour une protéase à sérine de $28 \mathrm{kD}$ appelée adipsine et non encore identifiée [17, 18].

La chronologie des événements décrits dans la figure 2 permet de rendre compte des observations faites sur le tissu adipeux in vivo et montrant que la diminution du nombre de cellules synthétisant l'ADN précède de peu l'augmentation du nombre de cellules possédant la glycérophosphate déshydrogénase et accumulant des triglycérides [19]. Cette chronologie présente également un intérêt direct pour l'étude du développement du tissu adipeux in vivo. En effet, aussi bien dans le cas de sa mise en place dans les divers sites au cours de la période post-natale que dans celui de l'hyperplasie à l'âge adulte, se posent les problèmes de la caractérisation des cellules précurseurs d'adipocytes et de l'estimation de leur nombre. Il faut rappeler que, à l'heure actuelle, seul le nombre des cellules contenant des triglycérides, et donc pleinement différenciées, peut être estimé. On conçoit que l'utilisation de marqueurs précoces, comme l'ARN messager pOb24, devrait permettre par hybridation in situ d'évaluer le" nombre de cellules dormantes qui, ayant exprimé le seul sousprogramme précoce de différenciation, resteraient présentes à l'âge adulte et seraient donc capables 
Tableau 1

LIGNÉES ÉTABLIES ET NON ÉTABLIES DE CELLULES PRÉCURSEURS D’ADIPOCYTES

\begin{tabular}{|c|c|c|}
\hline Lignées établies & Espèces & Origine \\
\hline 1246 & $\begin{array}{l}\text { Cellules de carcinome embryonnaire de } \\
\text { souris }\end{array}$ & Lignée T-948 \\
\hline$\left\{\begin{array}{l}\text { 3T3-L1 } \\
\text { 3T3-F442A } \\
\text { A31T }\end{array}\right.$ & $\begin{array}{l}\text { Embryon de souris Swiss } \\
\text { Embryon de souris Balb }\end{array}$ & $\begin{array}{l}\text { Variant de la lignée de fibroblastes } 3 \text { T3 } \\
\text { Variant d'une lignée de fibroblastes }\end{array}$ \\
\hline$\left\{\begin{array}{l}\text { Ob17 } \\
\text { HGFu }\end{array}\right.$ & $\begin{array}{l}\text { Souris adulte } \mathrm{C} 57 \mathrm{BL} / 6 \mathrm{~J} \text { ob/ob } \\
\text { Souris adulte } \mathrm{C} 57 \mathrm{BL} / 6 \mathrm{~J}+1 ?\end{array}$ & $\begin{array}{l}\text { Fraction adipocyte } \\
\text { de tissu adipeux épididymaire }\end{array}$ \\
\hline ST 13 & Souris ddN & Carcinome de glande mammaire \\
\hline MC3T3-G2/PA6 & Souriceau & Calvaria* \\
\hline MS3-2A & Souris adulte & Moelle osseuse ${ }^{*}$ \\
\hline BFC-1 & Souris adulte $\mathrm{C} 57 \mathrm{BL} / 6 \mathrm{~J}$ & $\begin{array}{l}\text { Fraction stroma-vasculaire de tissu adipeux } \\
\text { brun }\end{array}$ \\
\hline $\begin{array}{l}\text { Lignées non } \\
\text { établies }\end{array}$ & $\begin{array}{l}\text { Rat } \\
\text { Mouton } \\
\text { Bœuf } \\
\text { Porc } \\
\text { Homme } \\
\text { Souris } \\
\text { Rat } \\
\text { Homme }\end{array}$ & $\begin{array}{l}\text { Fraction stroma-vasculaire de tissu adipeux } \\
\text { épididymaire } \\
\text { Fraction stroma-vasculaire de tissu adipeux } \\
\text { sous-cutané et périrénal } \\
\text { Fraction stroma-vasculaire de tissu adipeux } \\
\text { sous-cutané } \\
\text { Fraction stroma-vasculaire de tissu adipeux } \\
\text { sous-cutané } \\
\text { Fraction stroma-vasculaire de tissu adipeux } \\
\text { omental et inguinal } \\
\text { Moelle osseuse* } \\
\text { Moelle osseuse } \\
\text { Moelle osseuse }\end{array}$ \\
\hline
\end{tabular}

* Les cellules de ces lignées, contrairement à celles des autres lignées, répondent aux corticostéroïdes pour l'accumulation des triglycérides mais ne répondent pas à l'insuline.

d'entrer dans la voie de la différenciation terminale en présence de stimuli mitogéniques et/ou adipogéniques appropriés.

Les changements profonds du métabolisme cellulaire qui accompagnent la différenciation ont conduit à rechercher des modifications du contenu intracellulaire et de la synthèse de certaines de ces enzymes spécifiquement induites. Dans tous les cas étudiés, l'augmentation d'une activité enzymatique est en corrélation avec une augmentation parallèle du contenu net de l'enzyme et de l'ARN messager correspondant. Il existe clairement une augmentation de $\mathrm{m} / \mathrm{s} n^{\circ} 7$ vol. 3 , seplembre 87 la transcription de gènes spécifiques de la différenciation adipocytaire [20-22] dont la séquence est connue pour trois d'entre eux [23-25]. On est en droit de penser que ces gènes présentent dans leur structure une ou plusieurs séquences impliquées dans la régulation de leur transcription. L'étude des gènes codant pour la protéine aP2 et la glycérophosphate déshydrogénase (induits de manière coordonnée) et du gène codant pour l'ARN messager de l'adipsine (induit plus tardivement) montre, en amont de la boîte TATA, l'existence de plusieurs copies d'une séquence con- sensus de 13 bases. Seuls les deux gènes exprimés de manière coordonnée possèdent également une séquence :

5'-ACTC(G)AGAGGAAAAG-3' située 118 bases (protéine aP2) et 183 bases (glycérophosphate déshydrogénase) en amont du site d'initiation de la transcription. Le gène complet $\mathrm{aP} 2$ inséré dans le plasmide pUC-18 peut s'exprimer lorsque les préadipocytes transfectés deviennent pleinement différenciés en cellules adipeuses; on peut ainsi envisager l'exploration systématique de l'expression d'un tel gène, délété d'une ou deux de ces séquences dont le rôle dans 
la régulation de la transcription pourrait donc être précisé.

\section{Rôle des hormones}

$\mathrm{La}$ régulation multihormonale s'exerce au niveau de l'expression des marqueurs tardifs. L'hormone somatotrope [26] et la triiodothyronine [27] présentes à concentrations physiologiques ont un effet direct et se sont révélées indispensables à la différenciation terminale des cellules de lignées établies, c'est-à-dire de cellules ayant déjà exprimé le sous-programme précoce [28]. Ces observations rejoignent celles faites sur l'animal et l'homme montrant que ces hormones sont nécessaires à l'acquisition d'un nombre adéquat de cellules adipeuses [29-32]. Les travaux les plus récents indiquent que l'exposition des cellules à l'hormone somatotrope pendant la phase d'émergence des marqueurs précoces, bien qu'elle ne soit pas nécessaire à l'expression de ces derniers, se révèle en revanche indispensable à l'expression des marqueurs tardifs. Le rôle de l'hormone somatotrope paraît multiple, dans la mesure où les cellules exposées (a) répondent mieux à l'action mitogène de
l'IGF-I [33], (b) synthétisent très rapidement l'ARN messager codant pour l'IGF-I et (c) voient leur niveau de spermidine intranucléaire augmenté, ce niveau étant corrélé de manière significative à l'expression des ARN messagers tardifs spécifiques de la différenciation adipocytaire [34] ; la régulation de l'expression des ARN messagers tardifs par l'hormone somatotrope se situe pour l'essentiel à un niveau transcriptionnel [28]. Les conclusions sur le rôle joué par l'insuline et les facteurs insulino-mimétiques (IGFs) restent partielles. Certes, le rôle modulateur positif mais non permissif que l'insuline joue sur l'expression des marqueurs précoces et surtout tardifs [35] est en accord avec les nombreuses données in vivo sur le contrôle de la synthèse des triglycérides et donc de l'hypertrophie des adipocytes. Toutefois, un rôle permissif pour l'expression de l'ARN messager codant pour la phosphoénolpyruvate carboxykinase a été récemment observé ; de même, un troisième rôle en tant que mitogène ne peut être exclu. En effet, dans ce dernier cas, les préadipocytes en culture primaire ayant déjà exprimé la lipoproprotease homologue. Proc Natl Acad Sci USA $1985 ; 82: 6480-4$

19. Cook JR, Kozak LP. sn-glycerol-3phosphate dehydrogenase gene expression during mouse adipocyte development in vivo. Dev Biol 1982 ; 92 : 440-8.

20. Djian P, Phillips M, Green $H$. The activation of specific gene transcription in the adipose conversion of 3 T3 cells. $J$ Cell Physiol 1985 ; 124 : 554-6.

21. Bernlohr DA, Bolanowski MA, Kelly $\mathrm{TJ} \mathrm{Jr}$, Lane D. Evidence for an increase in transcription of specific mRNAs during differentiation of 3T3-L1 preadipocytes. $J$ Biol Chem * $1985 ; 260$ : 5563-7.

22. Cook KS, Hunt CR, Spiegelman BM. Developmentally regulated mRNAs in 3T3-adipocytes : analysis of transcriptional control. J Cell Biol 1985 ; 100 : 514-20.

23. Ireland RC, Kotarski MA, Johnston LA, Stadler U, Birkenmeier E, Kozak L. Primary structure of the mouse glycerol-3-phosphate dehydrogenase gene. J Biol Chem 1986 ; 261 : 11779-85.

24. Phillips M, Djian P, Green H. The nucleotide sequence of three genes participating in the adipose differentiation of $3 \mathrm{~T} 3$ cells. $J$ Biol Chem 1986 ; 261 : 10821-7.

Figure 2. Les étapes de la différenciation adipocytaire. Ce schéma fait la synthèse des données obtenues in vitro et in vivo. La multiplication des cellules précurseurs (étapes 1 et 2) se produit in vitro sur une période de trois à cinq jours soit dans un milieu contenant du sérum ou du plasma, soit dans un milieu chimiquement défini contenant insuline, transferrine, FGF (" fibroblast growth factor ") et, selon les lignées préadipocytaires, une protéase à sérine de type kallikréine. La différenciation se déroule in vitro sur une période de une à deux semaines. Les cellules précurseurs ayant exprimé in vitro l'ARN messager pOb24 et l'ARN messager codant pour IGF-I (étape 4) sont également présentes in vivo dans la fraction stroma-vasculaire du tissu adipeux. L'ARN messager pOb24 diminue fortement in vitro (étapes 5 à 7) et est absent in vivo de la fraction contenant les adipocytes mûrs. Les ARN messagers codant pour la phosphoénolpyruvate carboxykinase (PEPCK) et pour l'adipsine s'expriment très tardivement in vitro (étape 7) et ne sont présents in vivo que dans la fraction contenant les adipocytes mûrs. Les résultats in vitro montrent que la présence de l'hormone somatotrope et de la triiodothyronine est obligatoire pour franchir les étapes 3 à 7. Ils montrent également que le processus d'expansion clonale (étapes 4 à 5) nécessite la stimulation simultanée des voies de production de l'AMP cyclique et de mobilisation des polyphosphoinositides ; cette étape s'accompagnerait d'une synthèse d'IGF-I et de la possibilité pour les cellules de répondre efficacement à ce mitogène. La réponse des cellules à l'insuline et aux $\beta$-adrénergiques augmente au cours des étapes 2 à 4. Toutefois, l'acquisition de la réponse lipolytique des cellules, qui permet l'hydrolyse des triglycérides cellulaires en acides gras, ne devient complète qu'au cours des étapes 5 et 6. 
ETAPE

Mutiofestion

Arrote de to

multiplication of

ecpusition des

merpurs pricoces

Acquistion de to

encitite de ipones

- IGF.I

Auprentation de la

ilpones wis hormones

potriges of 1

linsuline

Synthise $8 A D N$ of multiplication limitce correspondant un expansion clonale

Acovisition des athonotypes terdif's ot cecumbtion

Acoursition des

phenotypes ints tardifs

of eccumdation

\section{CELLULE \\ PRÉCURSEUR}

1

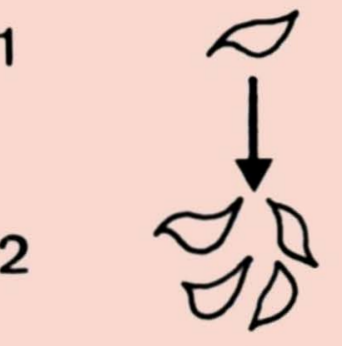

2

\section{PHÉNOTYPES}

APPARUS

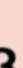

4
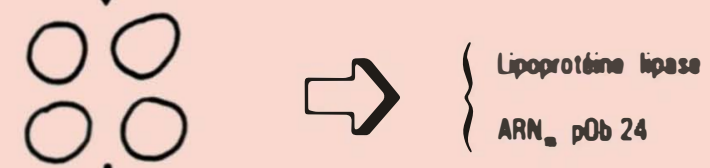

4<smiles>[O]</smiles><smiles></smiles>

5 ARN. IGF.I

5

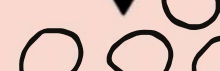<smiles>CCc1ccccc1CC</smiles>

000<smiles>c1ccc(-c2ccccc2)cc1</smiles>

Acoevy.CoA carboxylase ATP-citrate lyase

Enrme maliqu

Acito Gres syntherease

Glyctrophosphate deshydropdraseso Prottina AP2

Lipase hormono-sensitio

phosphocisstisase irts affin

6
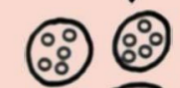

(8)

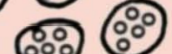

(ㅇ)
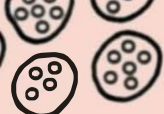

$\square$ pour rAMP cyclinge

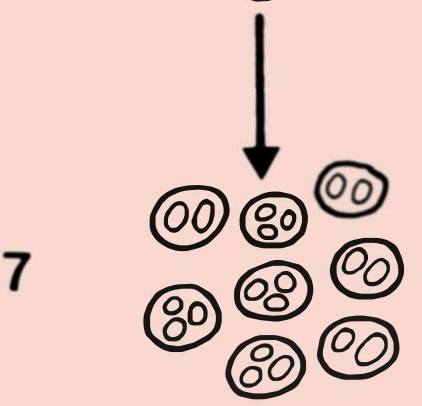
Acyl.CoA: Glyctrophosphate acyltranstitrese
Acyl.COA : Lysophosphatidase acyltranstitrase
AcytCoA : Didyctride acyltrensthese

$5\left\{\begin{array}{l}A R N_{0} \text { PEPCK } \\ A R N_{0} \text { ACDSin }\end{array}\right.$

CELLULLE ADIPEUSE 


\section{RÉFÉRENCES}

25. Hunt CR, Ro JHS, Dobson DE, Min HY, Spiegelman BM. Adipocyte P2 gene : developmental expression and homology of 5'-flanking sequences among fat cell-specific genes. Proc Nall Acad Sci USA 1986; 83 . 3786-90.

26. Morikawa M, Nixon T, Green H. Growth hormone and the adipose conversion of 3T3 cells. Cell $1982 ; 29$ : 783-9.

27. Grimaldi $P$, Djian $P$, Négrel $R$, Ailhaud G. Differentiation of Ob17 preadipocytes to adipocytes : requirements of adipose conversion factor(s) for fat cell cluster formation. $E M B O J 1982$; 6 : 687-92.

28. Doglio A, Dani C, Grimaldi P, Ailhaud $G$. Growth hormone regulation of the expression of differentiation-dependent genes in preadipocyte Ob1771 cells. Biochem $J 1986$; 238 : 123-9.

29. Levacher C, Sztalryd C, Kinebanyan M-F, Picon L. Effects of thyroid hormones on adipose tissue development in Sherman and Zucker rats. Am J Physiol 1984 ; 246 : C50-6.

30. Hollenberg $\mathrm{CH}$, Vost A. Regulation of DNA synthesis in fat cells and stromal elements from rat adipose tissue. $J$ Clin Invest $1968 ; 47: 2485-98$

31. Bonnet $F$, Vanderschueren-Lodeweyckx M, Beckels R, Malvaux P. Subcutaneous adipose tissue and lipids in blood in growth hormone deficiency before and after treatment with human growth homone. Pediatr Res $1974 ; 8: 800-5$.

32. Murakawa S, Raben MS. Effect of growth hormone and placental lactogen on DNA synthesis in rat costal cartilage and adipose tissue. Endocrinology $1968 ; 83$ : 645-50.

33. Zezulak KM, Green $\mathrm{H}$. The generation of insulin-like growth factor 1 - sensitive cells by growth hormone action. Science $1986 ; 233$ : $551-3$

34. Amri E, Barbaras R, Doglio A, Dani C, Grimaldi P, Ailhaud G. Role of spermidine in the expression of late markers of adipose conversion. Effects of growth hormone. Biochem $J$ $1986 ; 239$ : 363-70.

35. Vannier C, Gaillard D, Grimaldi P, et al. Adipose conversion of $\mathrm{Ob} 17$ cells and hormone-related events. Int $J$ Obes 1985 ; 9 : $41-53$.

36. Chapman A B, Knight DM, Ringold GM. Glucocorticoid regulation of adipocyte differentiation : hormonal triggering of the developmental program and induction of a differentiation-dependent gene. J Cell Biol $1985 ; 101: 1227-35$. téine lipase, peuvent en présence d'IGF-I répondre à l'insuline par une prolifération limitée avec, comme attendu, une expression des marqueurs tardifs [11]. Le rôle joué par les glucocorticoïdes, les androgènes et les estrogènes a fait jusqu'à présent l'objet de conclusions variées et contradictoires. Il apparaît cependant que les glucocorticoïdes, sans être indispensables, amplifient notablement l'expression du programme de différenciation de préadipocytes de la lignée établie TAI, cultivés en présence de sérum [36].

\section{Voies de recherche}

On ignore à l'heure actuelle si l'acquisition de nouveaux adipocytes, en particulier à l'âge adulte, est uniquement due à la différenciation terminale de cellules dormantes ayant déjà exprimé les marqueurs précoces, ou à la multiplication de précurseurs non différenciés. Dans la première hypothèse, la question reste posée de savoir si l'étape limitante de la différenciation terminale se trouve sous contrôle de stimuli mitogéniques et/ou adipogéniques. In vitro, un événement décisif paraît se situer au niveau de la multiplication limitée des cellules ayant exprimé le sous-programme précoce. En effet, la stimulation de la production d'AMP cyclique et la mobilisation simultanée des polyphosphoinositides se révèlent nécessaires pour que les cellules Ob17 possédant déjà la lipoprotéine lipase effectuent une seule division et acquièrent tous les phénotypes tardifs, à la condition que l'hormone somatotrope, la triiodothyronine et l'insuline soient présentes. Aucune autre hormone n'est alors requise. Ces résultats récents montrent ainsi que le processus de différenciation terminale se trouve in vitro sous la dépendance de plusieurs stimuli mitogéniques et adipogéniques. En tout état de cause, l'importance relative de ces différents stimuli dans la différenciation terminale de cellules dormantes reste à évaluer in vivo

\section{Summary}

Recent developments in obesity research have shown that the differentiation in vitro of adipose precursor cells from various species is a multi-step and coordinated process. This process has been mainly delineated with rodent preadipocytes from established cell lines. Early events are triggered by growth arrest and characterized by the emergence of a specific set of phenotypes detected both at the mRNA and protein level. The next critical event is a limited proliferation of these committed cells followed by their terminal, hormonally-controled differentiation, during which late and very late specific phenotypes appear and triglyceride accumulate. Studies in vitro show that the expression of late and very late specificdifferentiation genes is under the control of growth hormone and is regulated primarily at a transcriptional level.

Early and late phenotypes are detected in adipose tissue during adult life in the stromal-vascular fraction containing adipocyte precursors and in the fraction containing mature adipocytes, respectively. This suggests that the acquisition of new adipocytes in vivo might result from terminal differentiation of dormant (already committed) cells when exposed to appropriate stimuli. The complete characterization of these mitogenic and adipogenic stimuli is underway in vitro but remains to be settled in vivo.

\section{TIRÉS A PART}

G. Ailhaud : laboratoire de biologie du développement du tissu adipeux, centre de biochimie (Cnrs LP 7300), faculté des sciences, parc Valrose, 06034 Nice Cedex. 